\title{
The Role of HDACs Inhibitors in Childhood and Adolescence Acute Leukemias
}

\author{
Riccardo Masetti, Salvatore Serravalle, Carlotta Biagi, and Andrea Pession \\ Pediatric Oncology and Hematology "Lalla Seràgnoli” Unit, Department of Pediatrics, University of Bologna, 40137 Bologna, Italy \\ Correspondence should be addressed to Riccardo Masetti, riccardo.masetti@gmail.com
}

Received 11 July 2010; Revised 15 November 2010; Accepted 9 December 2010

Academic Editor: Christian Seiser

Copyright () 2011 Riccardo Masetti et al. This is an open access article distributed under the Creative Commons Attribution License, which permits unrestricted use, distribution, and reproduction in any medium, provided the original work is properly cited.

Acute leukemia is the most common type of childhood and adolescence cancer, characterized by clonal proliferation of variably differentiated myeloid or lymphoid precursors. Recent insights into the molecular pathogenesis of leukemia have shown that epigenetic modifications, such as deacetylation of histones and DNA methylation, play crucial roles in leukemogenesis, by transcriptional silencing of critical genes. Histone deacetylases (HDACs) are potential targets in the treatment of leukaemia, and, as a consequence, inhibitors of HDACs (HDIs) are being studied for therapeutic purposes. HDIs promote or enhance several different anticancer mechanisms, such as apoptosis, cell cycle arrest, and cellular differentiation and, therefore, are in evidence as promising treatment for children and adolescents with acute leukemia, in monotherapy or in association with other anticancer drugs. Here we review the main preclinical and clinical studies regarding the use of HDIs in treating childhood and adolescence leukemia.

\section{Introduction}

Acute leukemia is a malignancy characterized by clonal proliferation of variably differentiated myeloid or lymphoid precursors. It represents the most common type of childhood and adolescence cancer, accounting for $32 \%$ of all tumours diagnosed in children under 15 years of age and $26 \%$ of those diagnosed in adolescents under 20 years [1]. Over the past few decades, the prognosis of children and adolescents with acute leukemia has greatly improved, because of advances in risk assessment, tailored chemotherapy, optimal recourse to hematopoietic stem cell transplantation and innovative supportive care [2]. The cure rates now exceed $80 \%$ for children with acute lymphoblastic leukemia (ALL) and $50 \%$ for those with acute myeloid leukemia (AML) [3-5]. However, up to $20 \%$ of children with ALL and even more children with AML relapse, and these patients eventually present poor clinical outcome [6, 7]. Moreover a large number of patients presents several toxic side effects resulting from the very intensive chemotherapy [8]. Thus, novel therapeutic approaches are needed to increase the cure rate and the life's quality of these young patients.
In the past decade the increased understanding of the biology of acute leukemia, together with the implementation of high-throughput genomic techniques, have led to the development of molecular targeted therapies [9]. Epigenetic modifications, such as deacetylation of histones and DNA methylation, play crucial roles in the pathogenesis of many cancers including leukemia, by transcriptional silencing of critical genes [10]. The acetylation status of histones influences the chromatin conformation and consequently the transcription of genes. In normal cells there is a fine balance between acetylation and deacetylation of histones, depending on the activity of histone acetyl-transferases and histonedeacetylase (HDAC) [11] being the alteration of these enzymes possibly associated with tumorigenesis [12]. There are 18 HDACs in humans, 11 are zinc-dependent and fall into 4 classes on the basis of homology to yeast HDACs; the others are not zinc-dependent and not inhibited by compounds that inhibit zinc-dependent deacetylases [13]. HDACs has been reported in association with acute promyelocytic leukemia (APL) [14], and subsequently with many other hematologic and solid tumours [15]. Several studies have shown aberrant recruitment of HDAC-containing transcriptional repressor 
complexes by the fusion proteins PML-RAR $\alpha$ or PLFZ-RAR $\alpha$ and AML1-ETO in APL and AML, respectively, [16-19]. Furthermore, the constitutive upregulation of HOX genes that characterized AML with the mutation of the MLL gene has been shown to involve HDACs [20]. These observations led to the identification of HDACs as potential targets in the treatment of leukaemia. As a consequence, inhibitors of HDACs (HDIs) are being studied for therapeutic purposes, in an effort to upregulate the expression of the epigenetically silenced genes, potentially modifying the leukemic phenotype. HDIs promote apoptosis, cell cycle arrest and cellular differentiation, preventing malignant transformation [21, 22]. They are divided into several structural classes including short-chain fatty acids (such as valproic acid and butyrates), hydroximates (such as vorinostat and trichostatin-A), cyclic tetrapeptides (such as trapoxin and depsipeptide), benzamides (such as MS-275) and many other compounds [23]. In the last few years, studies of HDIs have shown that histone iperacetylation can be achieved safely in humans [21]. Thanks to the promising initial results obtained in adults with myelodisplastic syndrome and acute leukemia [24, 25], clinical trials are starting to involve pediatric patients too. In the future the use of HDIs, in monotherapy or in association with other anticancer drugs, could be a promising treatment for children and adolescents with acute leukemia. Here we review the main preclinical and clinical studies regarding the use of HDIs in treating childhood and adolescence leukemia.

\section{Preclinical and Clinical Trials}

Although only a few targeted agents are currently in general use, the number of preclinical and clinical studies of HDIs continue to grow. The trials of HDIs involving children and adolescents with acute leukemia are reported below. Molecule's structures, chemical class of the molecules and development status are resumed in Table 1.

Vorinostat (Suberoylamilide Hydroxamic Acid, SAHA). It is a hydroxamic acid multi-HDI that blocks the enzymatic activity of both Class I (HDAC1, -2, and -3) and Class II (HDAC6) HDACs at low nanomolar concentrations (IC50 < $86 \mathrm{nM}$ ) by directly binding to the catalytic site of these enzymes [26]. The anticancer potential of vorinostat was first demonstrated in nude mice transplanted with human prostate tumors [27]. Recently it has been approved by the U.S. Food and Drug Administration for the treatment of Cutaneous T-Cell Lymphoma (CTCL) and is now being evaluated also in other hematological malignancies [28].

Through its inhibitory action on HDACs, vorinostat has numerous effects on biological processes including cell cycle progression, apoptosis and differentiation at the cellular level, as well as angiogenesis inhibition and immune response modulation at the tissue level [26]. One model for the antitumor action of SAHA is that its inhibition of HDAC activity, and subsequent accumulation of acetylated histones, leads to the activation of genes whose expression causes induction of differentiation or apoptosis, thus inhibiting tumor growth. This model is based on the finding that the expression of a relatively small number of genes $(2-10 \%$ of expressed genes) is regulated following exposure of tumor cells to vorinostat [26]. One of the most commonly induced genes is the cyclin-dependent kinase inhibitor p21. However, rather than promoting apoptosis in tumor cells, it appears that vorinostat induced expression of p21 causes a cell cycle arrest in a p53-independent manner [29]. Moreover, increased acetylation of transcription factors such as p53, HIF- $1 \alpha$, and E2F and increased acetylation of cytoplasmic proteins such as $\alpha$-tubulin, cortactin, and HSP90 also contribute to vorinostat-induced cell cycle arrest, induction of cell death, and the inhibition of tumor growth [26].

Using two NOD/SCID mouse models, Einsiedel et al. demonstrated in vivo the efficacy of vorinostat in B-cell precursors childhood ALL. In fact, vorinostat was administered in a dose of 50,100 or $150 \mathrm{mg} / \mathrm{kg}$, in both subcutaneous and intravenous models, for 21 days and caused a clear growth suppression of the xenograft tumors [30].

As a multi-HDI, vorinostat may be well-suited for combination with other antineoplastic agents and radiation therapy, due both to its relatively low toxicity to normal cells and to its specific biological effects on cellular processes. Studies of the activity of vorinostat in combination with other cancer therapies (including radiation, kinase inhibitors, cytotoxic agents, and differentiating agents) have shown synergistic and additive activity in a variety of cultured human transformed cell lines [26]. Leclerc et al. showed that coadministration of SAHA plus the highly effective antileukemic drug methotrexate (MTX) synergizes to induce apoptotic death in ALL cells. When used as single agents, both MTX and SAHA can induce cell death, growth arrest and apoptosis, furthermore MTX plus SAHA synergistically increase apoptotic cell death and decrease viability in all B-precursor-ALL (SupB15, REH, NALM6, RCH-ACV) and T-ALL (CCRF-CEM) cell models tested [31]. Moreover, in vitro studies showed that SAHA causes growth inhibition, apoptosis, and potentiates retinoic acid induced differentiation in APL cells harboring $\mathrm{t}(15 ; 17)$ (NB4 cell line) and in BM cells from PLZF-RAR $\alpha / R A R \alpha-$ PLZF leukemic mice [32]. In the same study, He et al. demonstrated for the first time in vivo that SAHA induces an accumulation of acetylated histones at a nontoxic dose and, in combination with RA, prolongs survival and induces complete remission in APL transgenic mice refractory to retinoid acid treatment [32].

A phase I study of vorinostat in adult patients with leukemias was recently reported [33]. Significant antileukemia activity was observed in patients with AML, and vorinostat effectively inhibited HDAC activity in peripheral blood and bone marrow blasts. Further phase I and II trials of vorinostat as monotherapy or in combination regimens in adult AML are ongoing [33]. Moreover, a phase I study of vorinostat in pediatric patients with leukemia or solid tumors has just been published [34]. The primary purposes of this trial were to study the pharmacokinetics, the maximum tolerated dose (MTD) and the side effects of vorinostat in young patients (from 1 to 21 years old) and to determine the antitumor activity of SAHA administered with or without 13 -cis retinoic acid in these patients. 
TABLE 1: Main HDIs evaluated as emerging drugs for acute leukemias in pediatric and adult patients.

\begin{tabular}{|c|c|c|c|}
\hline Compound & Structure & Chemical class & Development status \\
\hline Vorinostat (SAHA) & & Hydroxamic acid & Phase II \\
\hline Valproic Acid & & Short-chain fatty acid & Phase II \\
\hline Entinostat (MS-275) & & Benzamide & $\begin{array}{l}\text { Preclinical and Phase } \\
\text { I only in adults }\end{array}$ \\
\hline $\begin{array}{l}\text { Pivaloyloxymethyl } \\
\text { butyrate (AN-9) }\end{array}$ & & Butyric acid & Preclinical \\
\hline $\begin{array}{l}\text { Givinostat } \\
\text { (ITF2357) }\end{array}$ & & Hydroxamic acid & Preclinical \\
\hline $\begin{array}{l}\text { Depsipeptide or } \\
\text { Romidepsin } \\
\text { (FK228) }\end{array}$ & & Cyclic tetrapeptide & Phase I only in adults \\
\hline $\begin{array}{l}\text { Mocetinostat } \\
\text { (MGCD0103) }\end{array}$ & & Benzamide & Phase I only in adults \\
\hline $\begin{array}{l}\text { Panobinostat } \\
\text { (LBH589) }\end{array}$ & & Hydroxamic acid & Phase I only in adults \\
\hline
\end{tabular}

This pediatric trial established the MTD of vorinostat as $230 \mathrm{mg} / \mathrm{m}^{2} / \mathrm{d}$ administered orally in patients with recurrent or refractory solid tumors, while patients with refractory leukemia did not appear to tolerate this dose due to liver dysfunction. Overall the authors pointed out that vorinostat drug disposition and tolerance in children was similar to that observed in adult patients. Furthermore, other two phase II studies are reported as currently recruiting participants. The first [35] is a trial studying the efficacy of the use of decitabine and vorinostat together with combination chemotherapy 
in treating patients with relapsed/refractory ALL or lymphoblastic lymphoma. They are recruiting patients from 2 to 60 years of age. The second study [36] is a clinical research study whose aim is to find the highest safe dose of vorinostat that can be given in combination with idarubicin and ara$\mathrm{C}$ for the treatment of AML and high-risk MDS. They are recruiting patients from 15 to 65 years of age.

Valproic Acid (VPA). It is a short-chain fatty acid that is a weak HDI, but its long term availability as antiepileptic drug prompted its evaluation in oncology as an epigenetic acting drug [28]. VPA can induce in vitro differentiation of primary AML blasts [37] and seems to exert its anticancer activity by inducing proteosomal degradation of HDAC, in particular of HDAC2 [38]. Furthermore, in $\mathrm{t}(8 ; 21)$ AML, VPA might target AML1/ETO-driven leukemogenesis through the disruption of aberrant HDAC1 function [19]. In fact, it has been reported that VPA treatment disrupts the AML1/ETO-HDAC1 physical interaction, stimulates the global dissociation of AML1/ETO-HDAC1 complex from the promoter of AML1/ETO target genes, and induces a significant inhibition of HDAC activity, histone $\mathrm{H} 3$ and $\mathrm{H} 4$ hyperacetylation, and recruitment of RNA polymerase II, leading to transcriptional reactivation of target genes otherwise silenced by AML1/ETO fusion protein. These pharmacological effects resulted in significant antileukemic activity mediated by partial cell differentiation and caspasedependent apoptosis [19]. Tonelli et al. showed that VPA induces strong cell growth inhibition in MLL-AF9 AML-M5 cells (THP-1, MM6 and MOLM-13) and AML-M5 blasts from a patient with a MLL gene rearrangement (MLLmut). This in vitro study indicated that VPA could activate a p53-independent G1 cell-cycle arrest and apoptosis in MLL-mut AML [39]. Also Einsiedel et al. demonstrated that VPA inhibits proliferation, induces apoptosis and histone $\mathrm{H} 4$ hyperacetylation in BCP-ALL cell lines (Reh, Nalm6, Z33) in vitro [30]. Moreover they showed that VPA administration reduce the tumor growth significantly in two NOD/SCID mouse models of BCP-ALL. VPA treatment was able to inhibit the leukemia-induced splenomegaly of animals after intravenous challenge with ALL blasts, while no apparent toxicity was detected [30]. Studies in mice with PML-RAR $\alpha$ induced leukemia demonstrated that VPA treatment causes upregulation of the death receptor proteins, TNF-related apoptosis inducing ligand (TRAIL), Fas, Fas ligand and DR5 in cells treated with VPA [40]. Also primary human AML samples that expressed neither PML-RAR $\alpha$ nor AML-ETO showed sensitivity to VPA and had increased apoptosis with evidence of upregulated TRAIL, DR5, Fas and Fas ligand levels in several cases that responded to HDAC therapy [40].

Several studies in vitro and in vivo showed that VPA is more efficacious in combination with other agents. Siitonen et al. investigated the effects of VPA as a single agent and in combination with cytarabine (Ara-C) and etoposide in an acute myeloblastic cell line (OCI/AML-2). They observed a dramatic increase in cytotoxicity combining VPA with Ara-C, whereas coaddition with etoposide had a much smaller effect on cell death. In addition, VPA induced a clear G1 phase arrest and up-regulated cyclin D1 expression in the presence of Ara-C and etoposide [41]. SanchezGonzalez et al. studied the cellular and molecular effects of combining the anthracycline idarubicin with VPA in HL60 and MOLT4 cell lines [42]. Their results indicate that the combination of an anthracycline with an HDI displays a synergistic effect in vitro and should have significant clinical activity in patients with leukemia [42]. Antileukemia activity has also been evaluated with VPA administered in combination with hypomethylating agents. The combination of 5-azacitidina (5-AZA), a hypomethylating agent with significant antileukemia activity in humans, with VPA was studied in the leukemic cell lines HL-60 and MOLT4. It showed a synergistic effect in growth inhibition, induction of apoptosis, and reactivation of protein p57 and p21 on human leukemia cell lines, suggesting that the combination of DAC and VPA could have significant antileukemia activity in vivo [43]. Several phase I/II studies of VPA monotherapy or in combination have been completed in adult patients. They include VPA plus decitabine [25], VPA plus retinoic acid (ATRA) [44, 45], and VPA plus 5-AZA and ATRA [46]. Garcia-Manero et al. conducted a phase $1 / 2$ study of the combination of decitabine and VPA in 54 patients with advanced leukemia and a median age at diagnosis of 60 years [25]. An objective response was observed in 22\% patients, including 19\% with a complete remission, and the overall survival was 15.3 months. This study enrolled also seven pediatric patients aged 4 to 21 years affected by relapsed/refractory AML. Although neither complete nor partial remission was described, 3 patients achieved a complete marrow response, and 1 patient presented only $6 \%$ of marrow blasts. No pediatric cases presented significant toxicities related to therapy [25]. Soriano et al. conducted a phase $1 / 2$ study of the combination of 5-AZA, VPA and ATRA in patients with AML or high-risk myelodysplastic syndrome [46]. The trial involved a total of 53 patients with a median age at diagnosis of 69 years. The combination of the drugs resulted safe and active, with an overall response rate of $42 \%$ and a median remission duration of 26 weeks. In their trial, Soriano and colleagues involved also 3 pediatric patients with recurring/refractory AML, and the combination of 5-AZA, VPA and ATRA led to BM response in 1 case [46]. Thus, combinations of VPA with 5-AZA or decitabine and ATRA need to be further explored in specific pediatric phase 1 studies in leukemia.

Entinostat (MS-275 or SNDX-275). It is a novel and orally available synthetic benzamide HDI that preferentially inhibits HDAC1, but does not possess activity against HDAC6 [47]. Rosato et al. conducted a study on MS-275 in human leukemia cell lines (U937, HL-60, Jurkat, and K562) and leukemic blasts obtained from the peripheral blood of patients with AML. They demonstrated that MS275 exerts dose-dependent effects on human leukemia cells: a p21-dependent growth arrest and differentiation at low drug concentrations and a marked induction of ROS, mitochondrial damage, caspase activation, and apoptosis at higher concentrations [48]. One study explored 
the effect of MS-275 against a panel of leukemia cells of human origin, each with defined genetic alterations. MS275 significantly induced growth arrest and apoptosis in the AML cell line, MOLM13, and in the biphenotypic leukemia cell line, MV4-11, which both possess an internal tandem duplication mutation in the FLT3 gene. MS-275 induced acetylation of HSP90 and ubiquitination of FLT3, which hesitated in the degradation of FLT3 via the proteasome pathway. Moreover, this study found that further inhibition of MEK/ERK signaling potentiated the effects of MS-275 in these cells. These results suggest that MS-275 may be useful in treating leukemic patients with a mutation on the FLT3 gene [49]. Another recent study showed that MS275 blocks Akt/mammalian target of rapamycin (mTOR) signaling in AML HL60 and APL NB4 cells, inducing growth arrest and differentiation. In addition, inactivation of mTOR by rapamycin analog RAD001 (everolimus) significantly enhanced MS-275-mediated growth inhibition and apoptosis of these cells and potentiated the ability of MS-275 to induce the differentiation of HL60 and NB4 cells. These results suggest that concomitant administration of an HDI and an mTOR inhibitor may be a promising treatment strategy for patients with AML or APL [50]. Moreover, MS275 might be used to enhance the antileukemic activity of established nucleoside analogues such as fludarabine. In fact Maggio et al. examined the interaction between MS-275 and fludarabine in lymphoid and myeloid human leukemia cells and demonstrated that sequential treatment of Jurkat lymphoblastic leukemia cells with MS-275 and fludarabine induces mitochondrial injury, caspase activation, and apoptosis [51]. The antitumor efficacy of MS-275 was also evaluated in vitro in a series of pediatric solid tumor cell lines, including neuroblastoma, rhabdomyosarcoma, Ewing's sarcoma (EWS), retinoblastoma, medulloblastoma, undifferentiated sarcoma (US), osteosarcoma, and malignant rhabdoid tumors and in vivo using xenograft orthotopic models of US, EWS, and neuroblastoma [52]. Jaboin et al. showed that MS-275 treatment of pediatric tumor cell lines induced the expression of p21 mRNA and different effects on cell cycle, like apoptosis, while drug administration in mice inhibited the growth of established tumors. Moreover, a phase I and pharmacologic study of MS-275 in adults with refractory and relapsed acute leukemias was reported by Gojo et al. [53]. They observed that the maximum-tolerated dose was $8 \mathrm{mg} / \mathrm{m}^{2}$ weekly for 4 weeks every 6 weeks and dose-limiting toxicities included infections and neurologic toxicity manifesting as unsteady gait and somnolence. They demonstrated that MS-275 effectively inhibits HDAC in vivo in patients, in fact the treatment induced an increase in protein and histone $\mathrm{H} 3 / \mathrm{H} 4$ acetylation, p21 expression, and caspase- 3 activation in bone marrow mononuclear cells.

Pivaloyloxymethyl Butyrate (AN-9). It is a relatively new member of an established family of acyloxyalkyl ester prodrugs of carboxylic acids that undergo rapid hydrolysis and its anticancer effect is assumed to stem primarily from the release of butyric acid [54]. AN-9 induces acetylation of histones at a concentration one order of magnitude lower than butyric acid in vitro and this is most likely due to its increased permeability across cell membranes [55]. Batova et al. investigated the in vitro therapeutic efficacy of AN-9 in HL-60 cell line and primary human acute leukemia cells isolated from bone marrow and the peripheral blood of pediatric patients with AML or B-precursor ALL, including doxorubicin-resistant and/or clinically refractory acute leukemias [54]. They demonstrated that AN-9 has antiproliferative and cytotoxic effects on all the leukemia cells tested, including a doxorubicin-resistant T-ALL, a clinically refractory relapsed AML, and a relapsed infant ALL characterized by an 11q23 rearrangement and a very poor prognosis. Furthermore, AN-9 is less toxic to normal hematopoietic progenitors and thus has selectivity for leukemia cells, indicating that AN-9 is a selective anticancer agent. The mechanism behind the antiproliferative effect of AN-9 appeared to be generally p21-independent and the increased apoptosis was thought to be mediated through the reduction in expression of the antiapoptotic bcl2 gene or alternatively through the induction of genes involved in the death receptor pathway [54]. Additionally, another advantage of AN-9 is its ability to synergize with anthracyclines such as daunomycin, commonly used in the treatment of leukemia patients, allowing the use of a lower dose of this drug and subsequently less toxicity. In fact Kasukabe et al. demonstrated that AN-9 is an effective antitumor agent in mouse monocytic leukaemia Mm-A cells and in human myeloid leukemia cell lines and that the administration of AN-9 plus daunorubicin markedly prolongs the survival of mice inoculated with Mm-A cells [56]. These several studies demonstrated the selective toxicity of AN-9 to acute leukemias, including drug-resistant relapsed leukemias, and thus provide the rationale for the initiation of clinical trials of AN-9 in acute leukemias of both adults and children. Futhermore three clinical trials of AN-9 in adults with others hematologic and solid tumours have been terminated, but the results have not yet been published. The first is a phase II study to assess the safety and efficacy of AN-9 in adult patients with chronic lymphocytic leukemia who have relapsed or refractory disease after previous chemotherapy treatment [57]. The second is a pilot phase I and II study of AN-9 in adult patients with malignant melanoma who have relapsed after treatment with chemotherapy or Interleukin2 [58]. The last is a phase II comparative trial of AN-9 and Docetaxel versus Docetaxel monotherapy in adult patients with advanced nonsmall cell lung cancer [59].

Givinostat (ITF2357). It is a synthesized HDI containing a hydroxamic acid moiety linked to an aromatic ring. It has been shown to inhibit the autonomous proliferation of haematopoietic cells bearing the JAK2(V617F) mutation, through a specific downmodulation of the JAK2(V617F) protein and inhibition of its downstream signalling [60]. Recently a phase II study has been reported in adult patients with JAK2V617F positive chronic myeloproliferative neoplasms [61]. Rambaldi observed that ITF2357 at doses of $50 \mathrm{mg}$ twice daily was well tolerated and could induce haematological response in most polycythemia vera and 
some myelofibrosis in adult patients carrying the JAK2 V617F mutation. In addition a phase II study testing givinostat in combination with hydroxyurea in a population of adult patients with JAK2V617F positive polycythemia vera nonresponders to the MTD of hydroxyurea monotherapy for at least 3 months is recruiting patients [62]. Furthermore ITF2357 was tested using an AML cell line as a model and it showed selective low-dose antileukemic activity on AML1/ETO-positive cells, emerging as a potent therapeutic agent, particularly in AML1/ETO-positive cells [63].

Depsipeptide (Romidepsin, FK228). It is a cyclic peptide that selectively inhibits HDAC isotypes 1, 2, 4 and 6 [28]. Recently it has been approved by the U.S. Food and Drug Administration for the treatment of Cutaneous T-Cell Lymphoma (CTCL) [64, 65] and is now being evaluated also in other hematological malignancies. Preclinical studies demonstrated that in vitro it inhibits HDAC in human APL cell line NB4, furthermore in vivo administration of depsipeptide alone partly inhibited the growth of established tumors of NB4 subcutaneously transplanted in NOD/Shiscid/scid mice and its combination with ATRA was synergistically effective in preventing leukemia death [66]. Depsipeptide was shown to inhibit HDAC in vivo in a phase I trial in adult patients with AML: several patients had evidence of antitumor activity following treatment, but no partial or complete responses were noted. Moreover its administration was limited by progressive adverse symptoms that prevented repeated dosing $[67,68]$. Therefore depsipeptide monotherapy appears to have limited clinical activity both in adult and pediatric patients.

Mocetinostat (MGCD0103). It is an isotype-specific aminophenylbenzamide that inhibits HDAC isotypes 1, 2, 3 and 11 [69]. Preclinical studies have demonstrated that MGCD0103 is orally bioavailable with significant in vitro antineoplastic activity at submicromolar concentrations against a broad spectrum of human cancers, including various leukemia cell lines and xenografts [70]. A phase I study in adult patients with AML demonstrated a favorable safety profile and showed activity as a single agent leading to a complete bone marrow response [69].

Several other HDIs have shown promise in adults in early phase I or small phase II trials. The hydroxamate panobinostat (LBH589) has attractive preclinical and phase I safety and efficacy profiles, with evidence of activity in AML [71]. Scuto et al. investigated the mechanism of action of LBH589 in two Philadelphia chromosome-negative ( $\mathrm{Ph}(-$ )) ALL cell lines (T-cell MOLT-4 and preB-cell Reh). Low nanomolar concentrations of LBH589 induced cell-cycle arrest, apoptosis, and histone hyperacetylation. LBH589 treatment also increased mRNA levels of proapoptosis, growth arrest, and DNA damage repair genes. This treatment was active against cultured primary $\mathrm{Ph}(-)$ ALL cells, including those from a relapsed patient, providing a rationale for exploring the clinical activity of LBH589 in treating patients with $\mathrm{Ph}(-)$ ALL [72]. A phase I study of LBH589 has been reported in adult patients with refractory hematologic malignancies [73]. Giles et al. observed that intravenous administration of LBH589 was well tolerated at doses $<11.5 \mathrm{mg} / \mathrm{m}^{2}$, while higher doses induced cardiac and other toxicities like nausea, diarrhea, vomiting, hypokalemia, loss of appetite and thrombocytopenia. Overall the drug treatment showed consistent transient antileukemic and biological effects that would be further explored using the oral formulation of LBH589.

\section{Conclusions and Perspectives}

In recent years HDIs have shown potential anticancer activity against a variety of solid and hematologic tumors, included leukemia. Vorinostat and depsipeptide have already been approved by the U.S. Food and Drug Administration for the treatment of cutaneous T-cell lymphoma [28, 65], and the clinical use of HDIs in adults with leukemia may become real in a short time. Several in vitro and in vivo studies have been performed to test the role of HDIs in leukemia treatments. The data from the adult trials show that HDIs, in monotherapy as well as in combination therapy, are generally well tolerated. However, since there are very few patients who have been treated for a long time, it will be necessary to monitor long-term effects in the future. However, the available data support the rationale for using HDIs in the treatment of childhood and adolescence leukemia. In the last few years, clinical trials of these epigenetic modulators have started to involve also children and adolescents affected by acute leukemia [25, 46]. Currently phase I-II clinical trials are evaluating the efficacy of vorinostat and VPA in treatment of childhood and adolescent leukemia, alone or in combination with other therapeutic agents [34-36]. In conclusion, further clinical trials involving HDIs are needed to consolidate the clinical use of these agents in both adult and pediatric acute leukemia. Moreover further studies are needed to determine whether specific cytogenetic or molecularly defined subgroups can predict response or resistance to HDIs therapy.

\section{References}

[1] Y. Ravindranath, "Recent advances in pediatric acute lymphoblastic and myeloid leukemia," Current Opinion in Oncology, vol. 15, no. 1, pp. 23-35, 2003.

[2] C. H. Pui, M. Schrappe, R. C. Ribeiro, and C. M. Niemeyer, "Childhood and adolescent lymphoid and myeloid leukemia," American Society of Hematology. Education Program, pp. 118$145,2004$.

[3] V. Conter, M. Aricò, G. Basso et al., "Long-term results of the Italian association of pediatric hematology and oncology (AIEOP) Studies 82, 87, 88, 91 and 95 for childhood acute lymphoblastic leukemia," Leukemia, vol. 24, no. 2, pp. 255264, 2010.

[4] M. Aricò, M. G. Valsecchi, C. Rizzari et al., "Long-term results of the AIEOP-ALL-95 trial for childhood acute lymphoblastic leukemia: insight on the prognostic value of DNA index in the framework of Berlin-Frankfurt-Muenster-based chemotherapy," Journal of Clinical Oncology, vol. 26, no. 2, pp. 283-289, 2008. 
[5] A. Pession, R. Rondelli, G. Basso et al., "Treatment and longterm results in children with acute myeloid leukaemia treated according to the AIEOP AML protocols," Leukemia, vol. 19, no. 12, pp. 2043-2053, 2005.

[6] G. Tallen, R. Ratei, G. Mann et al., "Long-term outcome in children with relapsed acute lymphoblastic leukemia after time-point and site-of-relapse stratification and intensified short-course multidrug chemotherapy: results of trial ALLREZ BFM 90," Journal of Clinical Oncology, vol. 28, no. 14, pp. 2339-2347, 2010.

[7] J. Abrahamsson, N. Clausen, G. Gustafsson et al., "Improved outcome after relapse in children with acute myeloid leukaemia," British Journal of Haematology, vol. 136, no. 2, pp. 229-236, 2007.

[8] G. J. L. Kaspers and C. N. Zwaan, "Pediatric acute myeloid leukemia: towards high-quality cure of all patients," Haematologica, vol. 92, no. 11, pp. 1519-1532, 2007.

[9] P. Brown, S. P. Hunger, F. O. Smith, W. L. Carroll, and G. H. Reaman, "Novel targeted drug therapies for the treatment of childhood acute leukemia," Expert Review of Hematology, vol. 2, no. 2, pp. 145-158, 2009.

[10] Y. Oki and J. P. Issa, "Review: recent clinical trials in epigenetic therapy," Reviews on Recent Clinical Trials, vol. 1, no. 2, pp. 169-182, 2006.

[11] G. Kouraklis and S. Theocharis, "Histone deacetylase inhibitors and anticancer therapy," Current Medicinal Chemistry, vol. 2, no. 4, pp. 477-484, 2002.

[12] S. Timmermann, H. Lehrmann, A. Polesskaya, and A. HarelBellan, "Histone acetylation and disease," Cellular and Molecular Life Sciences, vol. 58, no. 5-6, pp. 728-736, 2001.

[13] P. A. Marks and W. S. Xu, "Histone deacetylase inhibitors: potential in cancer therapy," Journal of Cellular Biochemistry, vol. 107, no. 4, pp. 600-608, 2009.

[14] M. A. Glozak and E. Seto, "Histone deacetylases and cancer," Oncogene, vol. 26, no. 37, pp. 5420-5432, 2007.

[15] R. P. Warrell Jr., L. Z. He, V. Richon, E. Calleja, and P. P. Pandolfi, "Therapeutic targeting of transcription in acute promyelocytic leukemia by use of an inhibitor of histone deacetylase," Journal of the National Cancer Institute, vol. 90, no. 21, pp. 1621-1625, 1998.

[16] R. J. Lin, L. Nagy, S. Inoue, W. Shao, W. H. Miller, and R. M. Evans, "Role of the histone deacetylase complex in acute promyelocytic leukaemia," Nature, vol. 391, no. 6669, pp. 811814, 1998.

[17] F. Grignani, S. De Matteis, C. Nervi et al., "Fusion proteins of the retinoic acid receptor- $\alpha$ recruit histone deacetylase in promyelocytic leukaemia," Nature, vol. 391, no. 6669, pp. 815$818,1998$.

[18] M. I. Klisovic, E. A. Maghraby, M. R. Parthun et al., "Depsipeptide (FR 901228) promotes histone acetylation, gene transcription, apoptosis and its activity is enhanced by DNA methyltransferase inhibitors in AML1/ETO-positive leukemic cells," Leukemia, vol. 17, no. 2, pp. 350-358, 2003.

[19] S. Liu, R. B. Klisovic, T. Vukosavljevic et al., "Targeting AML1/ETO-histone deacetylase repressor complex: a novel mechanism for valproic acid-mediated gene expression and cellular differentiation in AML1/ETO-positive acute myeloid leukemia cells," Journal of Pharmacology and Experimental Therapeutics, vol. 321, no. 3, pp. 953-960, 2007.

[20] A. M. Dorrance, S. Liu, W. Yuan et al., "Mll partial tandem duplication induces aberrant Hox expression in vivo via specific epigenetic alterations," Journal of Clinical Investigation, vol. 116, no. 10, pp. 2707-2716, 2006.
[21] M. Paris, M. Porcelloni, M. Binaschi, and D. Fattori, "Histone deacetylase inhibitors: from bench to clinic," Journal of Medicinal Chemistry, vol. 51, no. 6, pp. 1505-1529, 2008.

[22] A. Al-Janadi, S. R. Chandana, and B. A. Conley, "Histone deacetylation: an attractive target for cancer therapy?" Drugs in $R$ and D, vol. 9, no. 6, pp. 369-383, 2008.

[23] A. L. Abujamra, M. P. dos Santos, R. Roesler, G. Schwartsmann, and A. L. Brunetto, "Histone deacetylase inhibitors: a new perspective for the treatment of leukemia," Leukemia Research, vol. 34, no. 6, pp. 687-695, 2010.

[24] S. D. Gore, S. Baylin, E. Sugar et al., "Combined DNA methyltransferase and histone deacetylase inhibition in the treatment of myeloid neoplasms," Cancer Research, vol. 66, no. 12, pp. 6361-6369, 2006.

[25] G. Garcia-Manero, H. M. Kantarjian, B. Sanchez-Gonzalez et al., "Phase $1 / 2$ study of the combination of 5-aza-2'deoxycytidine with valproic acid in patients with leukemia," Blood, vol. 108, no. 10, pp. 3271-3279, 2006.

[26] V. M. Richon, J. Garcia-Vargas, and J. S. Hardwick, "Development of vorinostat: current applications and future perspectives for cancer therapy," Cancer Letters, vol. 280, no. 2, pp. 201-210, 2009.

[27] L. M. Butler, D. B. Agus, H. I. Scher et al., "Suberoylanilide hydroxamic acid, an inhibitor of histone deacetylase, suppresses the growth of prostate cancer cells in vitro and in vivo," Cancer Research, vol. 60, no. 18, pp. 5165-5170, 2000.

[28] N. Batty, G. G. Malouf, and J. P. J. Issa, "Histone deacetylase inhibitors as anti-neoplastic agents," Cancer Letters, vol. 280, no. 2, pp. 192-200, 2009.

[29] J. A. Vrana, R. H. Decker, C. R. Johnson et al., "Induction of apoptosis in U937 human leukemia cells by suberoylanilide hydroxamic acid (SAHA) proceeds through pathways that are regulated by $\mathrm{Bcl}-2 / \mathrm{Bcl}-\mathrm{x}(\mathrm{L})$, c-Jun, and $\mathrm{p} 21$ (CIP1), but independent of p53," Oncogene, vol. 18, no. 50, pp. 7016-7025, 1999.

[30] H. G. Einsiedel, L. Kawan, C. Eckert et al., "Histone deacetylase inhibitors have antitumor activity in two NOD/SCID mouse models of B-cell precursor childhood acute lymphoblastic leukemia," Leukemia, vol. 20, no. 8, pp. 1435-1436, 2006.

[31] G. J. Leclerc, C. Mou, G. M. Leclerc, A. M. Mian, and J. C. Barredo, "Histone deacetylase inhibitors induce FPGS mRNA expression and intracellular accumulation of longchain methotrexate polyglutamates in childhood acute lymphoblastic leukemia: implications for combination therapy," Leukemia, vol. 24, no. 3, pp. 552-562, 2010.

[32] L. Z. He, T. Tolentino, P. Grayson et al., "Histone deacetylase inhibitors induce remission in transgenic models of therapyresistant acute promyelocytic leukemia," Journal of Clinical Investigation, vol. 108, no. 9, pp. 1321-1330, 2001.

[33] G. Garcia-Manero, H. Yang, C. Bueso-Ramos et al., "Phase 1 study of the histone deacetylase inhibitor vorinostat (suberoylanilide hydroxamic acid [SAHA]) in patients with advanced leukemias and myelodysplastic syndromes," Blood, vol. 111, no. 3, pp. 1060-1066, 2008.

[34] M. Fouladi, J. R. Park, C. F. Stewart et al., "Pediatric phase I trial and pharmacokinetic study of vorinostat: a Children's Oncology Group phase I consortium report," Journal of Clinical Oncology, vol. 28, no. 22, pp. 3623-3629, 2010.

[35] M. J. Burke, Decitabine, Vorinostat, and Combination Chemotherapy in Treating Patients With Acute Lymphoblastic Leukemia or Lymphoblastic Lymphoma That Has Relapsed or Not Responded to Treatment, http://clinicaltrials.gov/ct2/ show/NCT00882206?term=VORINOSTAT+AND+leukemia\& rank $=6$. 
[36] G. Garcia-Manero, Phase II Study of Idarubicin, Cytarabine, and Vorinostat With High-Risk Myelodysplastic Syndrome (MDS) and Acute Myeloid Leukemia (AML), http:// clinicaltrials.gov/ct2/show/NCT00656617?term=VORINOSTAT+AND+leukemia\&rank $=20$.

[37] M. Göttlicher, S. Minucci, P. Zhu et al., "Valproic acid defines a novel class of HDAC inhibitors inducing differentiation of transformed cells," EMBO Journal, vol. 20, no. 24, pp. 69696978, 2002.

[38] O. H. Krämer, P. Zhu, H. P. Ostendorff et al., "The histone deacetylase inhibitor valproic acid selectively induces proteasomal degradation of HDAC2," EMBO Journal, vol. 22, no. 13, pp. 3411-3420, 2003.

[39] R. Tonelli, R. Sartini, R. Fronza et al., "G1 cell-cycle arrest and apoptosis by histone deacetylase inhibition in MLL-AF9 acute myeloid leukemia cells is p21 dependent and MLL-AF9 independent," Leukemia, vol. 20, no. 7, pp. 1307-1310, 2006.

[40] A. Insinga, S. Monestiroli, S. Ronzoni et al., "Inhibitors of histone deacetylases induce tumor-selective apoptosis through activation of the death receptor pathway," Nature Medicine, vol. 11, no. 1, pp. 71-76, 2005.

[41] T. Siitonen, P. Koistinen, and E. R. Savolainen, "Increase in Ara-C cytotoxicity in the presence of valproate, a histone deacetylase inhibitor, is associated with the concurrent expression of cyclin D1 and p27 in acute myeloblastic leukemia cells," Leukemia Research, vol. 29, no. 11, pp. 1335-1342, 2005.

[42] B. Sanchez-Gonzalez, H. Yang, C. Bueso-Ramos et al., "Antileukemia activity of the combination of an anthracycline with a histone deacetylase inhibitor," Blood, vol. 108, no. 4, pp. 1174-1182, 2006.

[43] H. Yang, K. Hoshino, B. Sanchez-Gonzalez, H. Kantarjian, and G. Garcia-Manero, "Antileukemia activity of the combination of 5-aza-2'-deoxycytidine with valproic acid," Leukemia Research, vol. 29, no. 7, pp. 739-748, 2005.

[44] A. Kuendgen, S. Knipp, F. Fox et al., "Results of a phase 2 study of valproic acid alone or in combination with all-trans retinoic acid in 75 patients with myelodysplastic syndrome and relapsed or refractory acute myeloid leukemia," Annals of Hematology, Supplement, vol. 84, no. 13, supplement, pp. 6166, 2005.

[45] G. Bug, M. Ritter, B. Wassmann et al., "Clinical trial of valproic acid and all-trans retinoic acid in patients with poor-risk acute myeloid leukemia," Cancer, vol. 104, no. 12, pp. 2717-2725, 2005.

[46] A. O. Soriano, H. Yang, S. Faderl et al., "Safety and clinical activity of the combination of 5-azacytidine, valproic acid, and all-trans retinoic acid in acute myeloid leukemia and myelodysplastic syndrome," Blood, vol. 110, no. 7, pp. 23022308, 2007.

[47] S. Minucci and P. G. Pelicci, "Histone deacetylase inhibitors and the promise of epigenetic (and more) treatments for cancer," Nature Reviews Cancer, vol. 6, no. 1, pp. 38-51, 2006.

[48] R. R. Rosato, J. A. Almenara, and S. Grant, "The histone deacetylase inhibitor MS-275 promotes differentiation or apoptosis in human leukemia cells through a process regulated by generation of reactive oxygen species and induction of p21CIP1/WAF1 1," Cancer Research, vol. 63, no. 13, pp. 36373645, 2003.

[49] C. Nishioka, T. Ikezoe, J. Yang, S. Takeuchi, H. Phillip Koeffler, and A. Yokoyama, "MS-275, a novel histone deacetylase inhibitor with selectivity against HDAC1, induces degradation of FLT3 via inhibition of chaperone function of heat shock protein 90 in AML cells," Leukemia Research, vol. 32, no. 9, pp. 1382-1392, 2008.

[50] C. Nishioka, T. Ikezoe, J. Yang, H. P. Koeffler, and A. Yokoyama, "Blockade of mTOR signaling potentiates the ability of histone deacetylase inhibitor to induce growth arrest and differentiation of acute myelogenous leukemia cells," Leukemia, vol. 22, no. 12, pp. 2159-2168, 2008.

[51] S. C. Maggio, R. R. Rosato, L. B. Kramer et al., "The histone deacetylase inhibitor MS-275 interacts synergistically with fludarabine to induce apoptosis in human leukemia cells," Cancer Research, vol. 64, no. 7, pp. 2590-2600, 2004.

[52] J. Jaboin, J. Wild, H. Hamidi et al., "MS-27-275, an inhibitor of histone deacetylase, has marked in vitro and in vivo antitumor activity against pediatric solid tumors," Cancer Research, vol. 62, no. 21, pp. 6108-6115, 2002.

[53] I. Gojo, A. Jiemjit, J. B. Trepel et al., "Phase 1 and pharmacologic study of MS-275, a histone deacetylase inhibitor, in adults with refractory and relapsed acute leukemias," Blood, vol. 109, no. 7, pp. 2781-2790, 2007.

[54] A. Batova, L. E. Shao, M. B. Diccianni et al., "The histone deacetylase inhibitor AN-9 has selective toxicity to acute leukemia and drug-resistant primary leukemia and cancer cell lines," Blood, vol. 100, no. 9, pp. 3319-3324, 2002.

[55] A. Aviram, Y. Zimrah, M. Shaklai, A. Nudelman, and A. Rephaeli, "Comparison between the effect of butyric acid and its prodrug pivaloyloxymethylbutyrate on histones hyperacetylation in an HL-60 leukemic cell line," International Journal of Cancer, vol. 56, no. 6, pp. 906-909, 1994.

[56] T. Kasukabe, A. Rephaeli, and Y. Honma, "An anti-cancer derivative of butyric acid (pivalyloxymethyl butyrate) and daunorubicin cooperatively prolong survival of mice inoculated with monocytic leukaemia cells," British Journal of Cancer, vol. 75, no. 6, pp. 850-854, 1997.

[57] A Pilot Study of Pivanex in Patients With Chronic Lymphocytic Leukemi, http://clinicaltrials.gov/ct2/show/NCT00083473? term $=$ AN-9\&rank $=1$.

[58] A Pilot Study of Pivanex in Patients With Malignant Melanoma, http://clinicaltrials.gov/ct2/show/NCT00087477? term=AN-9\&rank=2.

[59] Comparative Trial of Pivanex and Docetaxel Vs Docetaxel Monotherapy in Patients With Advanced NonSmall Cell Lung Cancer, http://clinicaltrials.gov/ct2/show/ NCT00073385?term=AN-9\&rank=3.

[60] V. Guerini, V. Barbui, O. Spinelli et al., "The histone deacetylase inhibitor ITF2357 selectively targets cells bearing mutated JAK2," Leukemia, vol. 22, no. 4, pp. 740-747, 2008.

[61] A. Rambaldi, C. M. Dellacasa, G. Finazzi et al., "A pilot study of the Histone-Deacetylase inhibitor Givinostat in patients with JAK2V617F positive chronic myeloproliferative neoplasms," British Journal of Haematology, vol. 150, no. 4, pp. 446-455, 2010.

[62] A. Rambaldi, Phase II Study of GIVINOSTAT (ITF2357) in Combination With Hydroxyurea in Polycythemia Vera (PV), http://clinicaltrials.gov/ct2/show/NCT00928707?term= NCT00928707\&rank=1.

[63] V. Barbetti, A. Gozzini, E. Rovida et al., "Selective antileukaemic activity of low-dose histone deacetylase inhibitor ITF2357 on AML1/ETO-positive cells," Oncogene, vol. 27, no. 12, pp. 1767-1778, 2008.

[64] R. L. Piekarz, R. Frye, M. Turner et al., "Phase II multi-institutional trial of the histone deacetylase inhibitor 
romidepsin as monotherapy for patients with cutaneous Tcell lymphoma," Journal of Clinical Oncology, vol. 27, no. 32, pp. 5410-5417, 2009.

[65] "StatBite: FDA oncology drug product approvals in 2009," Journal of the National Cancer Institute, vol. 102, no. 4, p. 219, 2010.

[66] H. Kosugi, M. Ito, Y. Yamamoto et al., "In vivo effects of a histone deacetylase inhibitor, FK228, on human acute promyelocytic leukemia in NOD/Shi-scid/scid mice," Japanese Journal of Cancer Research, vol. 92, no. 5, pp. 529-536, 2001.

[67] J. C. Byrd, G. Marcucci, M. R. Parthun et al., "A phase 1 and pharmacodynamic study of depsipeptide (FK228) in chronic lymphocytic leukemia and acute myeloid leukemia," Blood, vol. 105, no. 3, pp. 959-967, 2005.

[68] V. M. Klimek, S. Fircanis, P. Maslak et al., "Tolerability, pharmacodynamics, and pharmacokinetics studies of depsipeptide (Romidepsin) in patients with acute myelogenous leukemia or advanced myelodysplastic syndromes," Clinical Cancer Research, vol. 14, no. 3, pp. 826-832, 2008.

[69] G. Garcia-Manero, S. Assouline, J. Cortes et al., "Phase 1 study of the oral isotype specific histone deacetylase inhibitor MGCD0103 in leukemia," Blood, vol. 112, no. 4, pp. 981-989, 2008.

[70] M. Fournel, C. Bonfils, Y. Hou et al., "MGCD0103, a novel isotype-selective histone deacetylase inhibitor, has broad spectrum antitumor activity in vitro and in vivo," Molecular Cancer Therapeutics, vol. 7, no. 4, pp. 759-768, 2008.

[71] A. A. Lane and B. A. Chabner, "Histone deacetylase inhibitors in cancer therapy," Journal of Clinical Oncology, vol. 27, no. 32, pp. 5459-5468, 2009.

[72] A. Scuto, M. Kirschbaum, C. Kowolik et al., "The novel histone deacetylase inhibitor, LBH589, induces expression of DNA damage response genes and apoptosis in $\mathrm{Ph}$ acute lymphoblastic leukemia cells," Blood, vol. 111, no. 10, pp. 5093-5100, 2008.

[73] F. Giles, T. Fischer, J. Cortes et al., "A phase I study of intravenous LBH589, a novel cinnamic hydroxamic acid analogue histone deacetylase inhibitor, in patients with refractory hematologic malignancies," Clinical Cancer Research, vol. 12, no. 15, pp. 4628-4635, 2006. 

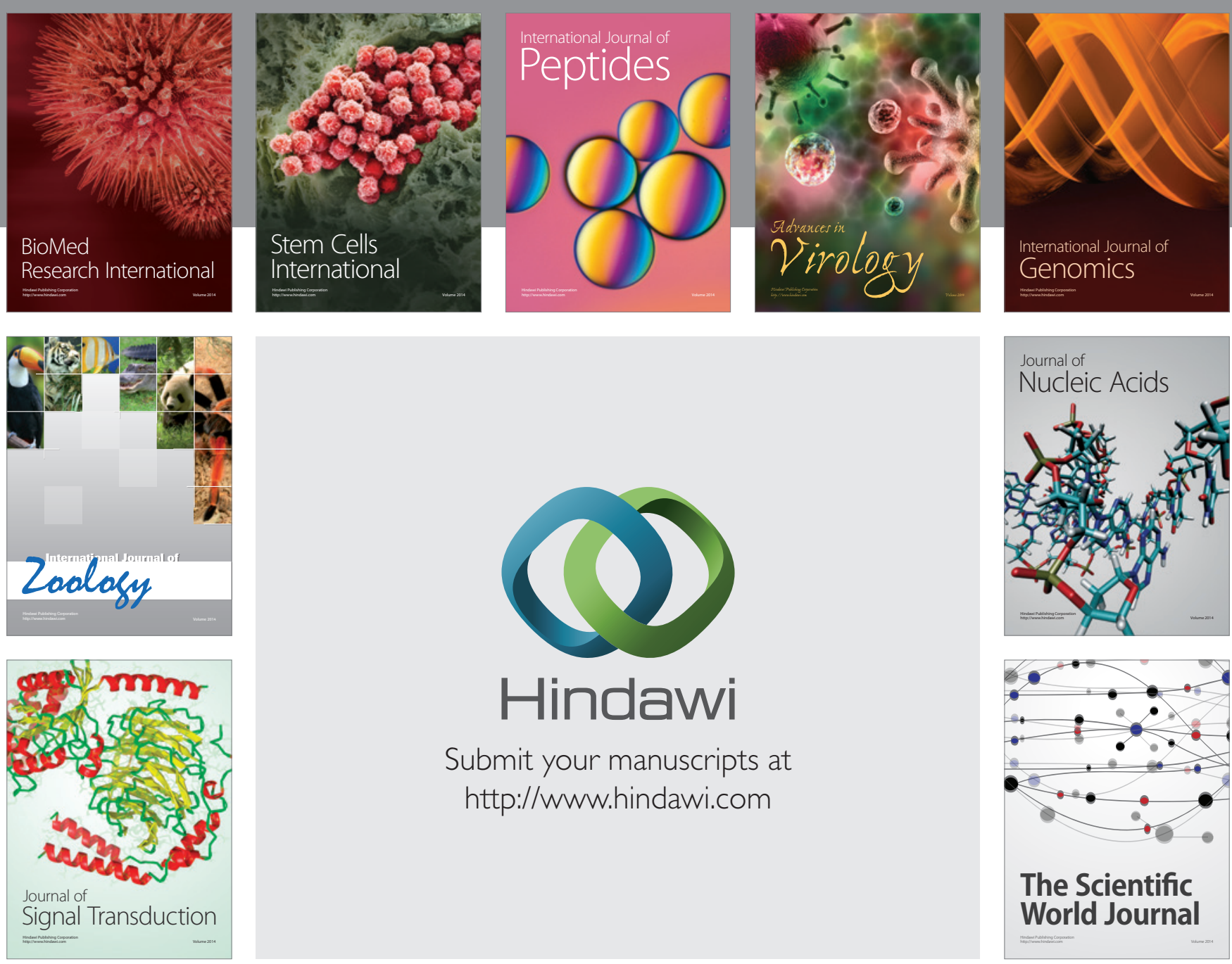

Submit your manuscripts at

http://www.hindawi.com
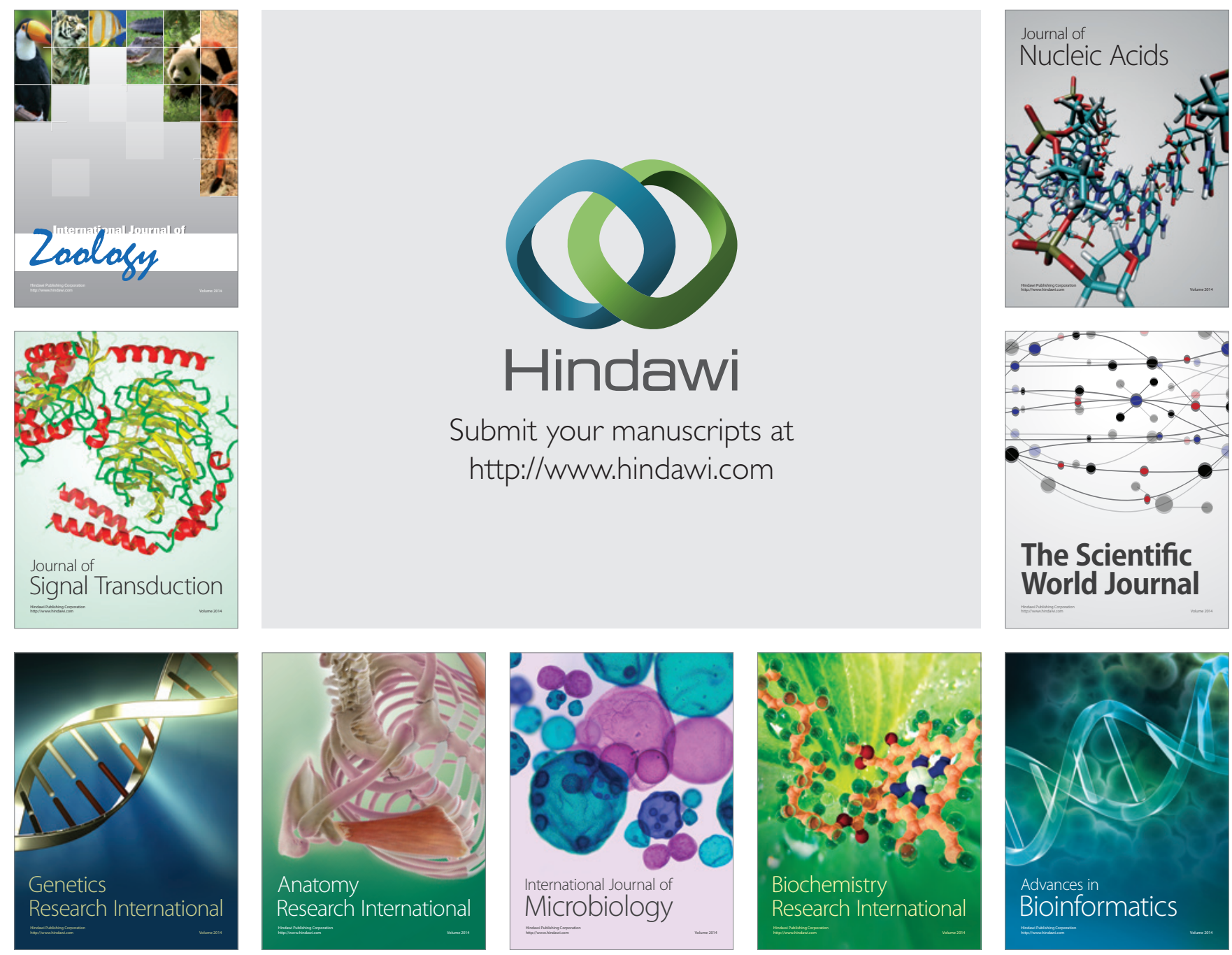

The Scientific World Journal
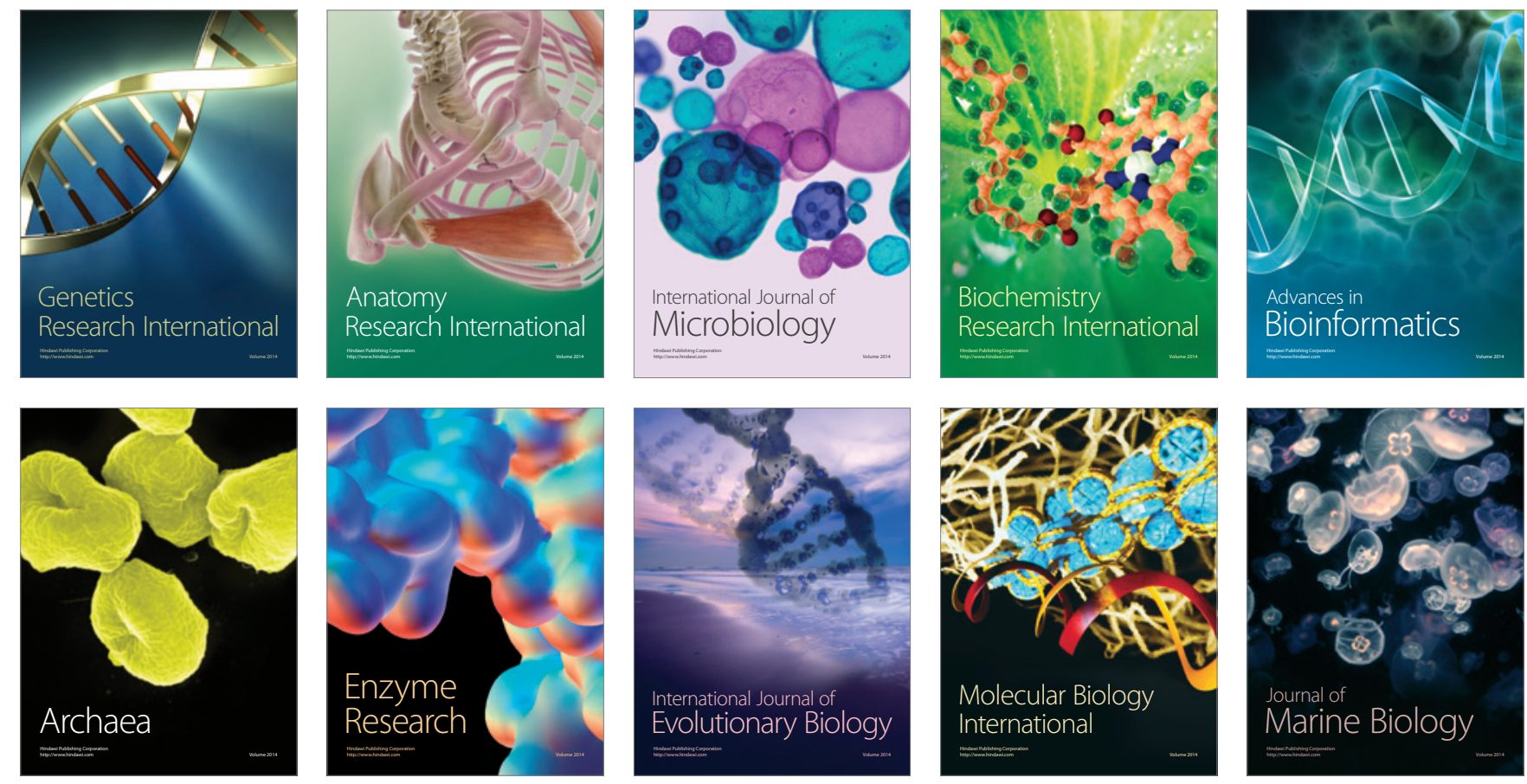